\title{
Bottom-up Specialisation of Logic Programs
}

\author{
Abstract \\ Wim Vanhoof, Danny De Schreye, and Bern Martens \\ Department of Computer Science, Katholieke Universiteit Leuven, \\ Celestijnenlaan 200A, B-3001, Heverlee, Belgium. \\ e-mail: $\{$ wimvh, dannyd, bern\}@cs. kul euven.ac. be
}

\section{Introduction and Motivation}

Partial deduction is an important transformation technique for logic programs, capable of removing inefficiencies from programs [4,5]. As an on-line specialisation technique, it is based on an evaluation mechanism for logic programs. The input to a typical partial deducer is a program and a partially instantiated query. The instantiated part represents the information with respect to which one would like to specialise; the uninstantiated part represents the information not yet known. Therefore, all classical partial deduction techniques use top-down evaluation (or SLD-resolution) to evaluate the program parts that depend on the known input and generate a new program that computes its result using only the remainder of the input. Since the new program has less computations to perform, in general, it will be more efficient.

In this work, we argue the need for a complementary partial deduction technique that is based on bottom-up evaluation of a logic program: starting from the unit clauses, information is propagated upwards, and new facts and clauses are derived. The motivation for such a complementary technique is twofold:

- It enables specialisation that is hard to achieve using a top-down approach. When specialising programs in which control information flows bottom-up, a top-down specialiser is often not capable of obtaining this information in time, in order to decide whether or not to continue the specialisation.

- Sometimes, one wishes to specialise (or "concretise") a program with respect to a set of definitions, providing information that flows bottom-up into the program. Top-down, goal-directed specialisation often is not the most natural approach to achieve this kind of specialisation.

To illustrate the control problem, consider the following example:

$$
\begin{aligned}
& \text { fill_list }(L, T, I, L) \leftarrow \operatorname{type}(T, L) \text {. } \\
& \text { fill_ist }(L, T, I, R) \leftarrow \text { fill_list }([I \mid L], T, I, R) \text {. } \\
& \text { type (list } 1,[X]) \\
& \text { make_list }(T, I, R) \leftarrow \text { fill_list }([], T, I, R) \text {. } \\
& \text { type (list } \left.3,\left[X_{1}, X_{2}, X_{3}\right]\right) \text {. }
\end{aligned}
$$

The predicate make list $(T, I, R)$ can be used to create a list of a fixed length (type $T$ ), with each element initialised with $I$. The result is returned in $R$. This example represents a class of recursive predicates that build up some structure 
between calls before a base clause of the predicate (ending the recursion) is reached, depending on the structure built.

Unfolding techniques, on which all top-down specialisers are based, are known to have problems with these. When unfolding e.g. make list (list3, $I, R$ ) without the information provided by the list-predicate, it is impossible to detect whether the sequence

$$
\text { fill_list }([], \ldots) \rightarrow \text { fill_list }([I], \ldots) \rightarrow \text { fill_list }([I, I], \ldots) \rightarrow \ldots
$$

will eventually terminate. If, on the other hand, this recursive predicate is handled in a bottom-up fashion, structure is shrinking between recursive calls, resulting in the facts

makedist (list $1, I,[I])$.

make_list(list3, $I,[I, I, I])$.

Apart from control, there are other reasons why a bottom-up approach might be preferred. Consider a program library $M$, in which $n$ predicates are defined, all using the functionality provided by an abstract data type (ADT) to hide the concrete representation and manipulation of a data structure. Despite the advantages of abstracting information through several layers during program development and maintenance, every such layer of abstraction decreases efficiency. Therefore, it makes sense to propagate the concrete representation up into the program, to the places where it is really used, thus removing the overhead. This sort of information propagation can in principle be obtained by a top-down specialisation scheme. Achieving it in a general and completely automatic way, however, is far from trivial, precisely because of the bottom-up flow of control information. In particular, (Vanilla-like) meta-programs typically present difficulties of this kind. Moreover, a top-down specialiser needs a single goal to start specialisation from, which might not be available when specialising a library. Or the goal(s) will likely contain no information (all arguments free) since the latter flows bottom-up (further complicating top-down control).

So, in a number of cases, proceeding bottom-up is a more natural solution. Bottom-up transformation and specialisation has been considered occasionally before (see e.g. [3]). However, to the best of our knowledge, our ongoing effort is the first attempt to achieve these in a completely general and automatic way.

\section{A Framework for Bottom-up Specialisation}

The most important contribution of this work, the details of which can be found in [7], is the definition of a formal framework, capturing the notion of bottom-up partial deduction.

The transformation is based on a non ground $T_{P}$-operator, $T_{P}^{C}$, acting on sets of clauses instead of atoms, as in compositional semantics [2,1]. In order to define a finitary transformation, the $T_{P}^{C}$-operator is combined with an abstraction function, mapping newly derived atoms (and clauses) onto more general clauses, capable of generating the former. This combination is denoted by an abstract 
$T_{P}^{C}$-operator, $A_{P}^{C}$. The resulting program can then be obtained from the least fixpoint of $A_{P}^{C}$, which can be ensured finitary through a suitable concrete control scheme. The transformation is proven sound and complete with respect to the S-semantics: transformation of a program $P$ results in a program $P^{\prime}$ having the same minimal S-Herbrand model [1] as $P$.

The defined transformation can be used as a stand-alone specialisation technique, useful when a program needs to be specialised with respect to its internal structure instead of a goal (as in the library-example). On the other hand, the bottom-up transformation can be combined with a more traditional topdown partial deduction strategy. In [6], we describe a concrete control scheme for bottom-up partial deduction, derived from top-down control techniques. As an illustration, it is shown that the Vanilla meta-interpreter can excellently be specialised by alternating a bottom-up transformation and a classical top-down specialisation - both using straightforward and fully automatic control. The same result can be obtained using a top-down component alone, but only at the cost of using a very sophisticated unfolding strategy. It is therefore expected that more involved combinations of bottom-up and top-down strategies will in general lead to specialisation techniques that are powerful, yet conceptually cleaner than one overall approach.

\section{Acknowledgments}

Wim Vanhoof is supported by a specialisation grant of the Flemish Institute for the Promotion of Scientific-Technological Research in Industry (IWT), Belgium. Danny De Schreye is a Senior Research Associate of the Belgian National Fund for Scientific Research and Bern Martens is partially supported by Esprit project 25503, ARGo.

\section{References}

1. A. Bossi, M. Gabbrielli, G. Levi, and M. Martelli. The S-semantics approach: Theory and applications. Journal of Logic Programming, 19/20:149-197, 1994.

2. A. Bossi, M. Gabbrielli, G. Levi, and M. C. Meo. A compositional semantics for logic programs. Theoretical Computer Science, 122(1-2):3-47, 1994.

3. Y. Cosmadopoulos, M. Sergot, and R. W. Southwick. Data-driven transformation of meta-interpreters: A sketch. In H. Boley and M. M. Richter, editors, Proceedings of the International Workshop on Processing Declarative Knowledge (PDK'91), volume 567 of LNAI, pages 301-308. Springer Verlag, 1991.

4. J. Gallagher. Specialisation of logic programs: A tutorial. In Proceedings PEPM'93, ACM SIGPLAN Symposium on Partial Evaluation and Semantics-Based Program Manipulation, pages 88-98, Copenhagen, June 1993. ACM Press.

5. J. W. Lloyd and J. C. Shepherdson. Partial evaluation in logic programming. Journal of Logic Programming, 11(3\&4):217-242, 1991.

6. W. Vanhoof, B. Martens, D. De Schreye, and K. De Vlaminck. Specialising the other way around. In J. Jaffar, editor, Proceedings of the Joint International Conference and Symposium on Logic Programming, Manchester, United Kingdom, June 1998. MIT-Press.

7. W. Vanhoof, D. De Schreye, and B. Martens. A framework for bottom up specialisation of logic programs. In Proceedings of the Joint International Symposia PLILP/ALP 1998, volume 1490 of Lecture Notes In Computer Science, pages 5472. Springer-Verlag, 1998. 